\title{
Induction of $\alpha 1$-Tubulin Gene Expression during Development and Regeneration of the Fish Central Nervous System
}

\author{
Virginia Hieber, Xinhua Dai, Mark Foreman, Daniel Goldman \\ Mental Health Research Institute and Department of Biological Chemistry, 205 Zina Pitcher Place, \\ University of Michigan, Ann Arbor, Michigan 48109
}

Received 28 April 1998; accepted 18 June 1998

\begin{abstract}
The $\alpha 1$ - and $\alpha 2$-tubulin encoding genes were cloned from a goldfish genomic DNA library. $\alpha 1-$ and $\alpha 2$-tubulin RNA expression was examined in developing and adult retinas. These studies demonstrated increased $\alpha 1$-tubulin RNA in presumptive ganglion cells that grow axons early in retinal development and in adult retinal ganglion cells whose optic axons had been damaged. The $\alpha 2$-tubulin RNA was undetectable in developing retina and constitutively expressed in adult retinal ganglion cells regardless of optic nerve crush. To determine if these changes in $\alpha 1$-tubulin RNA reflected changes in $\alpha 1$-tubulin promoter activity, we introduced into zebrafish embryos and adult goldfish retinal explants expression vectors harboring the $\alpha 1$ tubulin gene's promoter. These studies showed that the
\end{abstract}

$\alpha 1$-tubulin promoter confers a developmentally regulated, neuron-restricted pattern of reporter gene expression in vivo and its activity is increased in adult retinal neurons induced to regenerate their axons. Promoter deletions defined regions of $\alpha 1$-tubulin DNA necessary for this pattern of expression. These results suggest that DNA sequences necessary for $\alpha 1$-tubulin gene induction during central nervous system development and regeneration are contained within the $\alpha 1$-tubulin gene's 5' flanking DNA and that this promoter will be useful for identifying these elements and their DNA binding proteins. (๑) 1998 John Wiley \& Sons, Inc. J Neurobiol 37: 429-440, 1998

Keywords: CNS development; tubulin; gene expression; regeneration; zebrafish
The elaboration of an axon during nervous system development allows cells that are separated by relatively long distances to communicate with each other. In the adult, axon regrowth is a prerequisite to the reestablishment of appropriate connections and return of function following nerve damage. Although axon growth is necessary for establishing connections between cells during development and following nerve damage, very little is known about the molecular mechanisms mediating this process.

One approach to identifying these mechanisms is to identify proteins specifically necessary for axono-

Correspondence to: D. Goldman

Contract grant sponsor: Lucille P. Markey Charitable Trust

Contract grant sponsor: Glaucoma Foundation

(C) 1998 John Wiley \& Sons, Inc. CCC 0022-3034/98/030429-12 genesis. Some of these proteins would presumably be induced at times when the cell needs to generate an axon. Such proteins could then serve as probes for the mechanisms mediating their regulated expression, which would also contribute to successful growth of an axon.

One such protein is that encoded by the $\alpha 1$-tubulin gene. Tubulins are required for the formation of the cytoskeleton that accompanies axonal growth. Of all the $\alpha$-tubulins, the $\alpha 1$-isoform is specifically induced during development and regeneration of axons (Lewis et al., 1985; Miller et al., 1987, 1989; Skene, 1989; Gloster et al., 1994). Therefore, $\alpha 1$-tubulin expression may be a good probe for characterizing some of the mechanisms mediating axonal growth. Indeed, the creation of transgenic mice harboring the rat $\alpha 1$ tubulin (T $\alpha 1)$ promoter driving lacZ expression iden- 
tified a $1.1-\mathrm{kb}$ piece of T $\alpha 15^{\prime}$-flanking DNA that directed transgene expression to developing and regenerating neurons (Gloster et al., 1994).

We chose to examine $\alpha 1$-tubulin gene expression in zebrafish and goldfish because they offer several advantages over mammalian systems for studying axon growth during development and regeneration. First, unlike mammals, fish are able to mount a robust regenerative response following damage to their central nervous system (CNS). This is clearly demonstrated by the use of the goldfish visual pathway to successfully identify proteins that are induced during optic nerve regeneration and may participate in the successful reestablishment of retino-tectal connections (Grafstein, 1991; Murray and Grafstein, 1969; Heacock and Agranoff, 1982; Stuermer et al., 1992). Second, goldfish retina can be readily explanted into culture and extend neurites in response to an in vivo conditioning optic nerve crush (Landreth and Agranoff, 1976, 1979), facilitating studies of retinal ganglion cell axon regeneration. Third, zebrafish develop rapidly and are relatively transparent at early times of development when the nervous system is forming, facilitating visualization of neural structures (Ross et al., 1992). In addition, zebrafish embryos can be injected with DNA/RNA at the single-cell stage to investigate gene expression and protein function in vivo (Meng et al., 1997). With these advantages in mind, we chose to clone the $\alpha 1$-tubulin gene from goldfish and characterize its expression during nervous system development and regeneration. Our immediate aim is to determine if $\alpha 1$-tubulin gene expression in fish would serve as a suitable probe for unraveling mechanisms mediating neurite outgrowth in the developing and regenerating CNS. Our longterm goal is to use $\alpha 1$-tubulin gene expression as a probe for these mechanisms.

We report here that the $\alpha 1$-tubulin gene is linked to the $\alpha 2$-tubulin gene in the goldfish genome. RNase and in situ hybridization assays showed that $\alpha 1$-tubulin RNA is increased in retinal ganglion cells following optic nerve damage, while the $\alpha 2$ tubulin RNA is constitutively expressed. To determine if these changes in RNA expression are mediated by changes in $\alpha 1$-tubulin promoter activity, we cloned the $\alpha 1$-tubulin promoter and showed it is most active during CNS development and regeneration and that promoter deletions abrogate this activity. These results suggest the $\alpha 1$-tubulin promoter will be a useful probe for characterizing the mechanisms mediating CNS differentiation and regeneration in fish.

\section{MATERIALS AND METHODS}

\section{Goldfish Maintenance and Surgery}

Maintenance of goldfish and optic nerve crush were carried out as previously described (Hieber et al., 1992). Briefly, common goldfish (Carassius auratus) of 5-7 cm length were maintained at $25^{\circ} \mathrm{C}$ in well-aerated tanks. Right optic nerve crush was carried out just behind the orbit in tricaine methane sulfonate $(0.1 \%)$ anesthetized fish. The left optic nerve of each fish was left intact, with the left retina serving as an unoperated control.

\section{Isolation of Genomic $\alpha 1$ - and $\alpha 2$-Tubulin Clones}

A genomic goldfish library, cloned into the lambda dash vector (Stratagene), was kindly provided by Dr. Nisson Schecter (State University of New York at Stony Brook, Stony Brook, New York). Approximately $0.5 \times 10^{6}$ clones were screened with a radiolabeled goldfish $\alpha$-tubulin cDNA (clone 6 in Hieber et al., 1992). Three unique clones were purified. One of these clones, GF6, contained a 14.5-kb insert and was further characterized as reported in this article. DNA sequencing was performed using an Applied Biosystems automated DNA sequencer using thermal cycling procedures recommended by the manufacturer.

\section{RNase Protection Assays}

Total cellular RNA was isolated from goldfish retinas as previously described (Hieber and Goldman, 1993). RNase protection assays were performed as previously described (Chahine et al., 1993). The $\alpha 1$-tubulin probe is an antisense RNA corresponding to 49 bases of the $3^{\prime}$ end of the $\alpha 1$ tubulin coding sequence and extending into the $3^{\prime}$-untranslated sequence for an additional 251 nucleotides. The $\alpha 2$ tubulin probe is an antisense RNA corresponding to 98 bases of the $3^{\prime}$ end of the $\alpha 2$-tubulin coding sequence and extending into the $3^{\prime}$-untranslated sequence for an additional 245 nucleotides. ${ }^{32} \mathrm{P}$-radiolabeled RNA probes were prepared by run-off transcription of linearized vectors. The RNA probe was hybridized with $20 \mu \mathrm{g}$ of RNA overnight at $55^{\circ} \mathrm{C}$ and digested with RNase A for $1 \mathrm{~h}$, and the products were analyzed on a denaturing polyacrylamide gel. Probe integrity was confirmed by omitting RNase from the procedure. Controls consisted of using a sense strand probe, which consistently resulted in complete digestion of the probe.

\section{In Situ Hybridization}

Digoxygenin-labeled probes were prepared according to manufacturer's protocol (Boehringer Mannheim). The $\alpha 1$ and $\alpha 2$-tubulin probes both contain the last nine nucleotides of coding sequence from exon 4 and extend 3 ' into the untranslated region for an additional 341 and 231 nucleo- 
tides, respectively. Tissue sections were prepared and hybridized with digoxygenin-labeled probes (approximately 2 $\mu \mathrm{g} / \mathrm{mL}$ ) as previously described for radioactive probes (Hieber et al., 1992), except dithiothreitol (DTT) was omitted and tRNA $(10 \mu \mathrm{g} / \mathrm{mL})$ was included in the hybridization solution. Posthybridization treatments are identical to that previously reported through the RNase step (Hieber et al., 1992). Following RNase digestion slides were immersed in $0.5 \mathrm{M} \mathrm{NaCl}, 10 \mathrm{~m} M$ Tris- $\mathrm{HCl}(\mathrm{pH} 7.5), 1 \mathrm{~m} M$ ethylendiaminetetraacetic acid (EDTA) $(\mathrm{pH} 8)$ for $30 \mathrm{~min}$ at $55^{\circ} \mathrm{C}$ and then transferred to a solution of $2 \times \mathrm{SSC}, 1 \%$ nonfat dry milk, and $0.05 \%$ Triton X-100 and incubated for $2-3 \mathrm{~h}$ at $55^{\circ} \mathrm{C}$. Slides were then washed in buffer $1(0.1 \mathrm{M}$ Tris- $\mathrm{HCl}$ [pH 7.5], $0.15 \mathrm{M} \mathrm{NaCl}$ ) containing $1 \%$ nonfat dry milk and $0.05 \%$ Triton $\mathrm{X}-100$ for $30 \mathrm{~min}$ at room temperature. A $1 / 3000$ dilution of alkaline phosphatase coupled anti-digoxigenin antibody (Boehringer Mannheim) was prepared in buffer 1 containing $0.3 \%$ Triton X-100 and $1 \%$ nonfat dry milk. A few drops of this solution were added over sections and coverslipped. Slides were then placed in a humid chamber and incubated for $2-3 \mathrm{~h}$ at room temperature. Following the antibody incubation, slides were washed in buffer 1 for $30 \mathrm{~min}$ at room temperature (three changes) and then buffer 2 (0.1 $M$ Tris- $\mathrm{HCl}$ (pH 9.5), $0.1 M \mathrm{NaCl}, 50 \mathrm{mM} \mathrm{MgCl}{ }_{2}$ ) for $30 \mathrm{~min}$ at room temperature (three changes). Chromogen solution (buffer 2 containing $0.175 \mathrm{mg} / \mathrm{mL}$ 5-bromo-4chloro-3-indolylphosphate and $0.33 \mathrm{mg} / \mathrm{mL}$ nitroblue tetrazolium salt) was then applied to the slides and incubated for $2 \mathrm{~h}$ to overnight in the dark.

\section{Expression Vectors}

The $\alpha 1$-transcriptional start site was identified by an RACE polymerase chain reaction (PCR) protocol using goldfish RNA (Frohman, 1990) followed by cloning and sequencing of the amplified product. $\alpha 1$-Tubulin expression vectors were created by subcloning $\alpha 1$-tubulin $5^{\prime}$-flanking DNA in front of the green fluorescent protein (GFP) reporter of the pEGFP-1 vector (Clontech). The $\alpha 1$ TIpEGFP vectors contain various lengths of 5 -flanking DNA (designated by the number at the beginning of the name), exon 1, intron 1, and the first codon of exon 2 (amino acid 2 begins exon 2) fused in frame with the GFP sequence (see Fig. 5). 5' deletions were created by taking advantage of convenient restriction enzyme sites. pCS2 contains the cytomegalovirus promoter driving GFP expression.

\section{Retinal Explants and Electroporation}

The optic nerve emanating from the right retina was crushed in vivo. Seven days later, retinas were removed from darkadapted goldfish (we generally use 12 fish for each experiment) and placed in sterile phosphate-buffered saline (PBS). At this point, all subsequent procedures were carried out in a laminar flow hood. Retinas were washed with PBS and the peripheral outer third of the retina, containing undifferentiated precursor cells, was cut away and discarded. The remaining retina was cut into small pieces $(1-3 \mathrm{~mm})$ with scissors. Retinal pieces were washed four to five times with PBS and resuspended in electroporation buffer (100 $\mathrm{m} M$ Hepes [pH 7.4], $137 \mathrm{~m} M \mathrm{NaCl}, 6 \mathrm{~m} M$ dextrose, $4 \mathrm{~m} M$ $\mathrm{Na}_{2} \mathrm{HPO}_{4}$ ). We use $0.4 \mathrm{~mL}$ of electroporation buffer for two retinas. Then, $10 \mu \mathrm{g}$ of $\alpha 1$ TIpEGFP DNA and $40 \mu \mathrm{g}$ of BSSK (Stratagene) DNA was added to the $0.4 \mathrm{~mL}$ of retinal pieces in electroporation buffer. A BTX Electro Cell Manipulator 600 was used for electroporation. Settings were: resistance $=\mathrm{R} 6$; capacitance $=950 \mu \mathrm{F}$; and voltage $=326$ $\mathrm{V}$. Immediately following electroporation, the DNA/retinal suspension was transferred to a tissue culture dish containing L-15 media supplemented with 1\% FCS. Explants were maintained at room temperature in humidified chambers. Approximately 7 days later, retinal explants were examined using fluorescent microscopy to identify cells expressing the electroporated DNA. Quantitation of expression was performed by counting expressing cells in the explants. We found that electroporation resulted in numerous clumps of cells expressing the reporter gene (see Fig. 7 for an example) and the total number of cells comprising a clump was quite variable (1-100 cells). Regardless of whether we counted the total number of expressing cells or the total number of expressing clumps, we obtained similar results. Therefore, we reported our data as number of expressing cell clumps in right versus left retina.

\section{Zebrafish Injections}

Zebrafish embryos were obtained by natural crosses. Solution for DNA injection contained $50 \mathrm{ng} / \mu \mathrm{L} \alpha 1$ TIpEGFP in $250 \mathrm{~m} M \mathrm{KCl}$ and $0.1 \%$ Phenol red. Approximately $300 \mathrm{pL}$ of DNA solution was injected into one or two cell embryos with a glass micropipette. At $24-72 \mathrm{~h}$ postinjection, animals were anesthetized and examined using fluorescent microscopy. Pictures were taken of either developing fish or fish smashed under a coverslip. Quantitation of GFP expression was performed by counting the number of neural cells expressing GFP divided by the total number of cells expressing GFP in a microscopic field. Neural cells includes developing neurons and mature neurons and were defined as cells extending axons $>2$ cell diameters. Three different fields were counted and averaged. Cell counts were made using both whole-mount embryos and embryos smashed under a coverslip. Both methods of analysis gave similar results.

\section{RESULTS}

\section{$\alpha 1-$ and $\alpha 2-T u b u l i n$ Genes Are Linked in Goldfish Genome}

Although $\alpha 1$-tubulin gene expression has been shown to increase during development of the mammalian nervous system and upon regeneration of damaged peripheral neurons (Lewis et al., 1985; Miller et al., 1987, 1989; Gloster et al., 1994), it is not known if 
2.GF

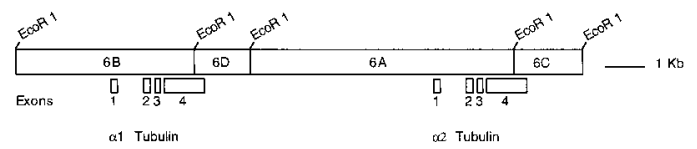

Figure 1 The $\alpha 1$ - and $\alpha 2$-tubulin genes are linked in the goldfish genome. A goldfish genomic library was screened with an $\alpha$-tubulin cDNA probe to isolate clone $\lambda \mathrm{GF} 6$. $\lambda \mathrm{GF} 6$ is approximately $14.5 \mathrm{~kb}$ and harbors the $\alpha 1$ - and $\alpha 2$-tubulin coding sequences in EcoRl restriction fragments $6 \mathrm{~B} / 6 \mathrm{D}$ and $6 \mathrm{~A} / 6 \mathrm{C}$, respectively. The open bar represents the genomic clone and the filled-in bars represent the location of the four exons comprising the $\alpha 1$ - and $\alpha 2$-tubulin RNAs.

this gene is regulated in a similar fashion in fish. Therefore, it was necessary to clone the fish $\alpha 1$ tubulin gene to provide a specific probe for $\alpha 1$-tubulin gene expression.

An $\alpha$-tubulin cDNA (Heiber et al., 1992) was used to isolate $\lambda \mathrm{GF} 6$ from a goldfish genomic library. $\lambda$ GF6 contains approximately $14.5 \mathrm{~kb}$ of insert DNA. Southern blotting and DNA sequencing identified tubulin coding sequences residing within this genomic clone. This analysis revealed that clone $\lambda \mathrm{GF} 6$ contains two $\alpha$-tubulin genes in tandem with their coding sequences residing in EcoRI fragments $6 \mathrm{~B} / 6 \mathrm{D}$ and 6A/6C, respectively (Fig. 1). Intron/exon boundaries were identified by comparison of genomic DNA with that of published tubulin cDNAs. Because 3'-untranslated sequences are not conserved between goldfish and other $\alpha$-tubulins, we did not define the exact $3^{\prime}$ boundary of exon 4 . However, both $6 \mathrm{~B} / 6 \mathrm{D}$ and $6 \mathrm{~A} / 6 \mathrm{C}$ tubulin genes contained a consensus polyadenylation signal sequence in exon 4 , which generally precedes the site of polyadenylation by about 10-30 nucleotides and provides an approximation of where exon 4 ends.

The deduced amino acid sequence for goldfish tubulins $6 \mathrm{~B} / 6 \mathrm{D}$ and $6 \mathrm{~A} / 6 \mathrm{C}$ revealed that they encode tubulin proteins that are identical to the mammalian $\alpha 1$ and $\alpha 2$ genes, respectively (Lemischka and Sharp, 1982; Cowan et al., 1983; Lewis et al., 1985; Ginzburg et al., 1986; Villasante et al., 1986). These two tubulins differ by a single amino acid at position 232 (Villasante et al., 1986).

\section{$\alpha 1-T u b u l i n$ RNA Increases during Development of the Retina}

We compared the expression of $\alpha 1$ - and $\alpha 2$-tubulin RNA in developing goldfish retina. $\alpha 1$ - and $\alpha 2$-specific probes were prepared from 3 '-untranslated sequences and incubated with stage 22 goldfish embryo retinal sections (Sharma and Ungar, 1980) for in situ hybridization assays. These assays showed increased $\alpha 1$-tubulin RNA in retinal cells dispersed throughout the central portion of the retina, with highest expression in those cells that are either approaching or
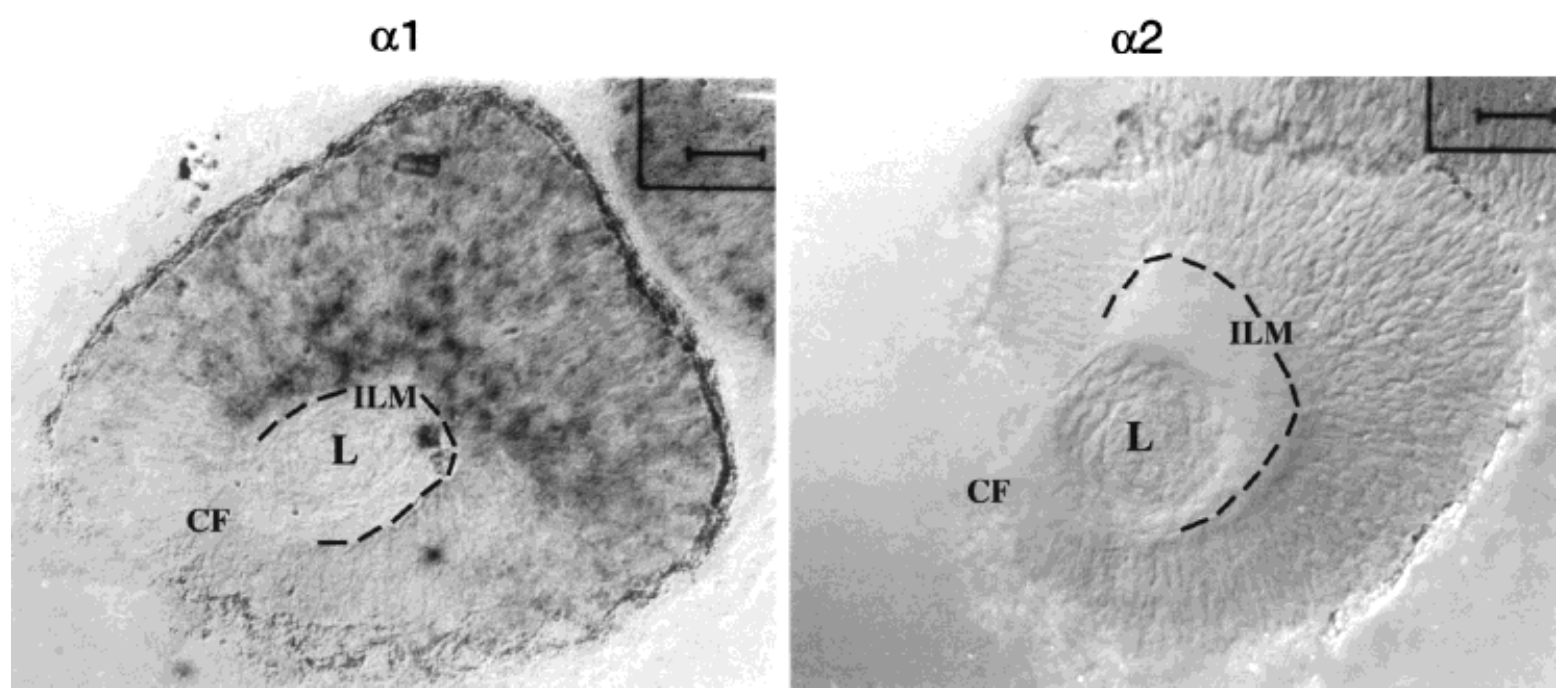

Figure 2 A specific induction of $\alpha 1$-tubulin RNA during retinal development. In situ hybridization was used to assay $\alpha 1$ - and $\alpha 2$-tubulin RNA levels during goldfish retina development. Stage 22 goldfish embryo (Sharma and Ungar, 1980) retinal sections (20 $\mu \mathrm{m}$ thick) were hybridized with $\alpha 1$ or $\alpha 2$-tubulin-specific probes. The dark reaction product in the panel labeled $\alpha 1$ identifies $\alpha 1$ tubulin RNA in the central portion of the retina, while no signal above background was detected with the $\alpha 2$-specific probe. $\mathrm{L}=$ developing lens; ILM = inner limiting membrane demarcated by a dashed line; $\mathrm{CF}=$ choroid fissure. Scale bar $=50 \mu \mathrm{m}$. 


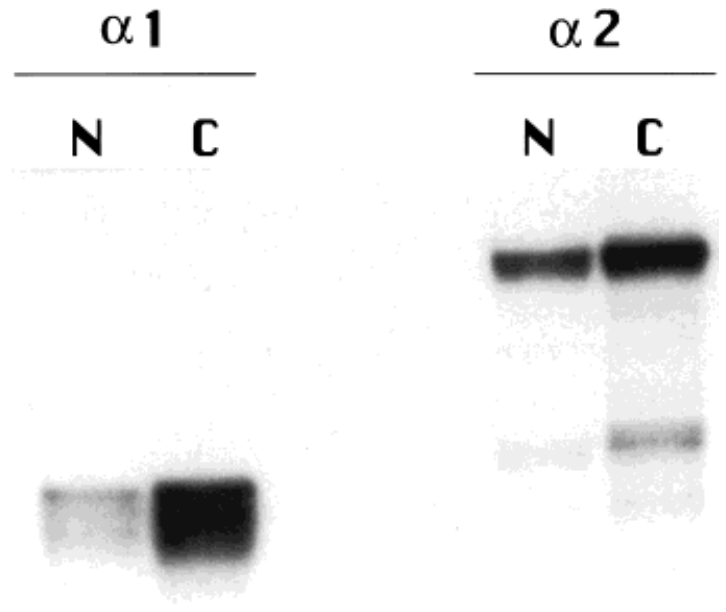

Figure 3 RNase protection assays identify a large increase in $\alpha 1$ RNA levels in retinas whose optic nerves are regenerating. The right optic nerve of adult goldfish was crushed and allowed to regenerate for 10 days, after which total retinal RNA was isolated from optic nerve crush (C) and normal (N) retina. $\alpha 1$ - and $\alpha 2$-specific 3 '-untranslated antisense probes were used to hybridize to RNA and RNaseresistant hybrids were resolved on denaturing polyacrylamide gels.

residing closest to the inner limiting membrane (Fig. $2)$. In contrast, $\alpha 2$-tubulin RNA expression was undetectable at this stage of retinal development (Fig. 2).

\section{$\alpha 1-T u b u l i n$ RNA Is Specifically Induced in Retinal Ganglion Cells upon Optic Nerve Regeneration}

RNase protection assays were performed to determine if the identified goldfish $\alpha 1$ - and $\alpha 2$-tubulin genes were expressed in adult retina and if their expression was regulated during optic nerve regeneration. For these experiments, we crushed the right optic nerve 10 days prior to isolating left and right retinas. Total retinal RNA was prepared and $\alpha 1$ - and $\alpha 2$-tubulin specific probes were used to quantitate their corresponding RNA levels. These experiments revealed a large increase in $\alpha 1$ RNA following optic nerve crush (Fig. 3). Quantitation by scanning densitometry identified a 10 -fold increase in $\alpha 1$-tubulin RNA, while the $\alpha 2$ RNA only increased twofold.

The above RNase protection assays average RNA levels across the entire retina and therefore may underestimate cell specific changes in RNA levels. To circumvent this problem, we used in situ hybridization assays. $\alpha 1$-Tubulin RNA was undetectable in normal goldfish retina (Fig. 4). However, by 10 days postoptic nerve crush, we observed a large increase in $\alpha 1$-tubulin RNA residing specifically in retinal ganglion cells. In contrast, $\alpha 2$-tubulin RNA appears to be constitutively expressed at a relatively low but detectable level, and, like the $\alpha 1$ RNA, $\alpha 2$ transcripts were selectively expressed in retinal ganglion cells.

\section{DNA Sequence of the Goldfish $\alpha 1$ - Tubulin Gene's 5'-Flanking Region}

The above experiments showed a specific increase in $\alpha 1$-tubulin RNA during retinal development and upon regeneration of damaged adult optic axons. This change in tubulin RNA level may represent transcriptional and/or posttranscriptional mechanisms. To explore the possibility that transcriptional mechanisms mediate changes in $\alpha 1$-tubulin RNA levels, we cloned and sequenced the $\alpha 1$ gene's 5'-flanking DNA.

Approximately $1.7 \mathrm{~kb}$ of DNA upstream from the $\alpha 1$ gene's transcription start site was sequenced (Fig. 5). Inspection of this sequence identified a number of putative regulatory elements (Fig. 5). Consensus TATAA and CCAAT boxes are located at position -28 and -71 , respectively. Upstream of these sequences are a variety of DNA elements that may participate in directing appropriate developmental and tissue-specific expression. These elements include a retinoic acid response element, E-box basic helixloop-helix consensus sequence, AP2 consensus sequence, and a homeodomain consensus motif for Drosophila antennapedia, fushi tarazu, and ultrabithorax homeodomain proteins (Fig. 5). In addition to these putative regulatory elements, we identified an AT repeat beginning at position - 1461 which is immediately followed by a GT repeat and a TAGA repeat beginning at position -1138 .

We also sequenced approximately $3 \mathrm{~kb}$ of DNA extending $5^{\prime}$ of the $\alpha 2$-tubulin gene's transcription start site and found little similarity to the $\alpha 1$ gene's 5'-flanking DNA (data not shown).

\section{The $\alpha 1$-Tubulin Promoter Restricts Reporter Gene Expression to the Developing Nervous System In Vivo}

To determine if the cloned $\alpha 1$-tubulin promoter contained sequences that directed expression to developing neurons in vivo, we injected zebrafish embryos with the $-1696 \alpha 1$ TIpEGFP expression vector. We chose to use an expression construct containing the $\alpha 1$-tubulin gene's first intron since it showed significantly higher expression in vivo than those constructs lacking the first intron. Injected fish were analyzed at 24,48 , and $72 \mathrm{~h}$ postinjection (data only shown for 


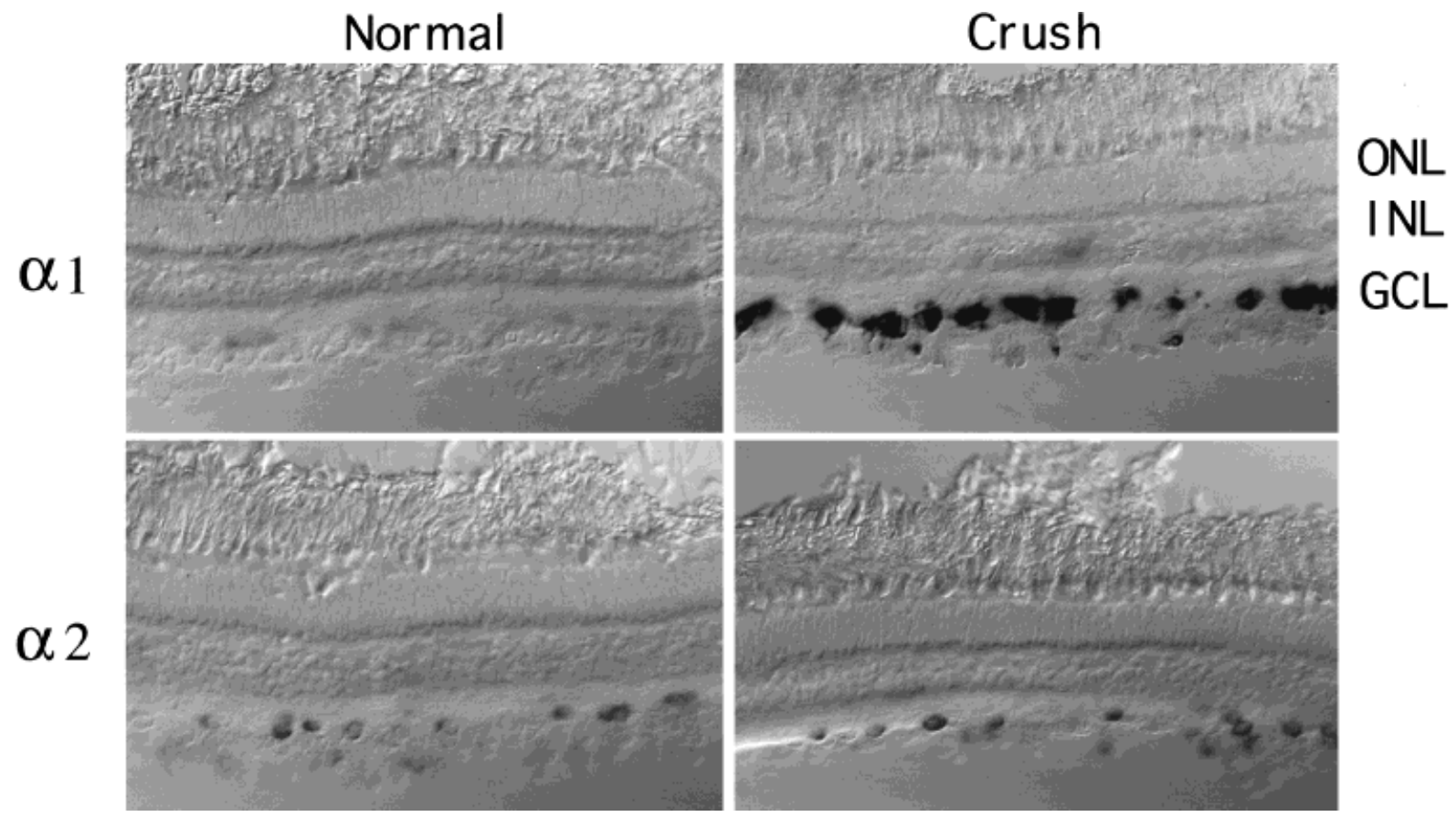

Figure 4 A specific induction of $\alpha 1$-tubulin RNA in retinal ganglion cells following optic nerve crush. Adult goldfish were induced to regenerate their right optic nerve by crushing the nerve emanating from the right retina. The left retina served as a normal control. Ten days following optic nerve crush, retinas were isolated and sectioned for in situ hybridization. $\alpha 1$ - and $\alpha 2$-tubulin specific probes were hybridized with left (normal) and right (crush) retinal sections. The $\alpha 1$ probe identified a large increase in $\alpha 1$-tubulin RNA following optic nerve crush that is specific to the retinal ganglion cells, while the $\alpha 2$-tubulin probe identified a low basal level of $\alpha 2$-tubulin RNA expression in both normal and optic nerve crush retina. ONL $=$ outer nuclear layer; INL $=$ inner nuclear layer; GCL $=$ ganglion cell layer. Scale bar $=100 \mu \mathrm{m}$.

$48 \mathrm{~h}$ ). This analysis revealed maximal transgene expression around $48 \mathrm{~h}$ postinjection. Expression patterns were mosaic with fish exhibiting as few as two expressing cells to well over 1000 expressing cells. At $48 \mathrm{~h}$ of development, $\alpha 1 \mathrm{TIpEGFP-injected} \mathrm{embryos}$ showed preferential and highest expression in cells extending axons [Fig. 6(A,B)]. GFP expressing cells could be found in retina, forebrain, mesencephalon, hindbrain, and spinal cord. Quantitation of this expression pattern (Table 1) showed that of those fish expressing GFP, $98 \%$ had at least some neural expression. Quantitation of the number of GFP-positive cells in these fish revealed that $82 \%$ of the expressing cells are neurons. This is in contrast to an expression vector harboring the CMV promoter driving GFP expression which showed widespread expression in most cell types of the developing zebrafish embryo, with only about $10 \%$ of the GFP expressing cells representing neurons [Fig. 6(C,D) and Table 1].

Unidirectional deletions of the $\alpha 1$-tubulin promoter identified regions of DNA that appear to contribute to a robust developmentally induced neuron-specific expression pattern (Table 1). First, deletion of approximately $900 \mathrm{bp}$ from the $5^{\prime}$ end of the $\alpha 1$ promoter $(-808 \alpha 1$ TIpEGFP) resulted in a small $(15 \%)$ reduction in the number of embryos exhibiting neural-specific expression and a similar decrease in the percentage of GFP cells that are neural. However, deletion of an additional $500 \mathrm{bp}$ $(-302 \alpha 1$ TIpEGFP) caused a $19 \%$ decrease in the number of embryos showing neural expression and also reduced the percentage of GFP-expressing cells that are neurons to $42 \%$. However, this expression pattern is still neurally restricted compared to the promiscuous expression driven by the CMV promoter which only resulted in about $10 \%$ of the total GFP-expressing cells representing neurons. Finally, deletion of an additional $120 \mathrm{bp}$ $(-182 \alpha 1$ TIpEGFP) dramatically reduced the number of embryos exhibiting expression in the nervous system (10\%) and the percentage of total GFPexpressing cells representing neurons $(8 \%)$. The pEGFP vector without a promoter showed no significant expression. 


\begin{abstract}
-1696 TCCTGTAAGg TATATGAAAG CATTATtATT CTAAACATGT CCAAGgagCt TUTATGAGACC TGTCTTCACT GTTGGACTGT TGTTAGCAAC CAGCCTATAA

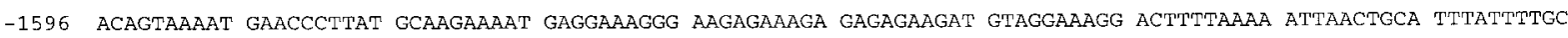

1496 ATTTATCAGT TGCAATATGA TGTTTAAAAT GCACGTATAT ATATATATAT ATATATATAT ATATATATGT GTGTGTGTGT GTGTGTGTGT GTGTGTGTGT

-1396 GTGTGTGTGT GTGTGTAATg AGTGGCTCAA AAAATATATT CAATATATTC TGGAAAACAA TGTTTTTGGA CTCACATAAA ATGTACTAGA AGAAATGTAC

-1296 CTTTATCCTT ATAATATTGC ATTTTATTGT GTTCATACTA TCAGAACTCT CAGTTTCAGT GCCTGTGATG AAGCCAAATA CTGAAAATTG TCATACTGAA

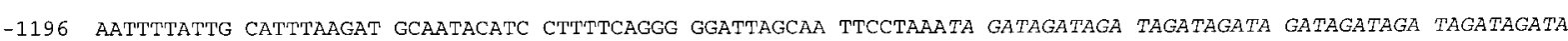

- 1096 GATAGATAGA TAGATAGATA GatAgATAGA TAGATAGATA GATAGAAGAC CTGGTGTTTA AAAGGTTGGg CCTGCTCCTC ATTCCCTGCT GGGGAaGCTG

-996 CATCCTCCCC CCTGAgTGCT GCTATTCAGA TTTGGGgTCA TGTGATCTAT GGCCGTTAat GTAGgCTTGT GTATTATCTC CAGGATACTA GTCAGTATCC

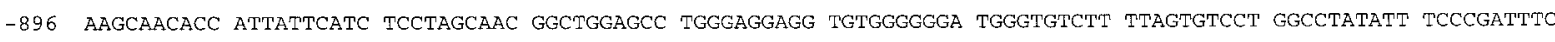

-796 CTCAACGTCT ATGCA $\overline{\text { GGGTC }}$ ATFTCACCTC GTACATTTAT GACTCAATAT TTTAAATGTC CATGTTCATA TAAATCCTTT ACATTTTTAC ACAGTCTTAC

-696 ATCTTAATCT GTCCACCTCA GCAGATTCAT CCCTGTAGCT CATTTGAAAT TGGTTCACGT GCAACTTGGT TAAACTATAG AATAATCCCT CTCTTAGTTA

-596 TCCTTCGGAG CACAAAGATA ACAGTTTAAA GAATATGTGA CAACTTACTC CAGAATTTAC CCCAGGCAAA AGGGGCAGAA GAAGAGAaCA GATGGAGAAT

-496 ATGCTGATCA AGATACACAA AGATGCTGGT ACATACTGTA AGGTTCAGAT GTTATAdAAT AAAATAATAA TAATAATAAT AATAAATCCT GATGCATGGT

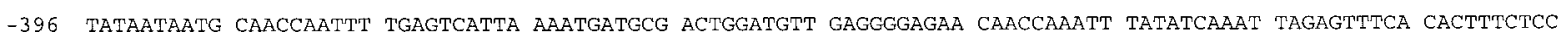

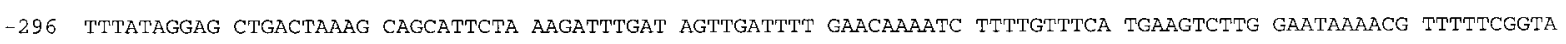

-196 TGATTCAGTG TTTCAGATCT ATTGgGTGAg GTTCTGGgag GTGTCTAAAT AACCCTAATC TTCCTGACAA CCAATAGAAA TCAGTCTAGC CCTTCGAACT

-96 GGGCAGATTT CATCGCGTCT GACCAATCAA AGGTAAGAAA CGTGGTCGCC TGGGCAACAC TCATATATAA AGTGAGGTCA GTCCGTTATA ACGTTATTCT

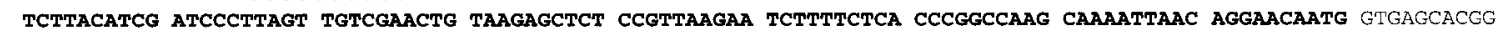

105 TTTCATATAG CCTAATTATT GATTGCTTAT TTAAATAACC TTTTTTATTC GACTAGCTTT TAAAGTATCT ATATTTAACT AATATTTCGg GTATATAAAA

205 TATAGTTTTA TAAAGAaCAT ATTCATTTAA AGAAACGATT TAGTATACGT ATTAAAAAAA GAAAAAAACG CTTTGGTTTT GTAAATGATG TATTTTGAAT

305 TATT'TAAAA TPAATTIGTG CATTTAAAAT ATATTTTATA CAAATAATGT AGTCACGGTT GTGCTTATAA CCTAAAAAAA GAATATGGAA AAATAAGAGG

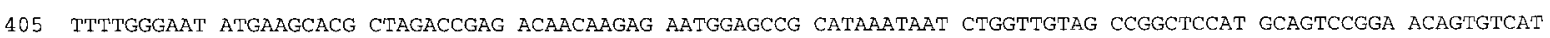

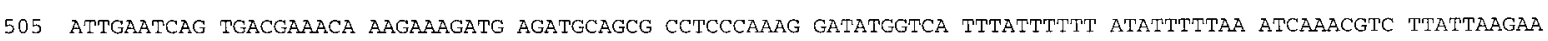

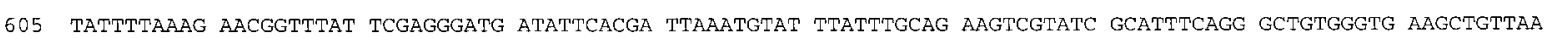

705 ATATTCATGG TGATGTAGAC GGCATCACTT ATGCAGCTGA TGGACATCAC ATTACCAGTA AaCCAGCAGT TGATTGTGTT ATGTGATCGC TTCTTCTGAC

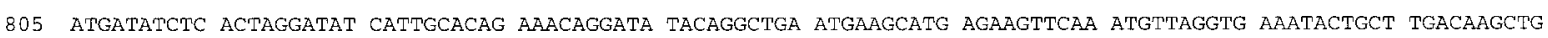

905 AagCTTTACA TGTGACTGAA ATTTACATGC TGCAATRATG TTTGTTTGGT TTGACTTTTA ACTTTTGCCT TTTTCTTCAC AGCGT
\end{abstract}

Figure 5 DNA sequence of the $\alpha 1$-tubulin gene's 5'-flanking DNA, exon 1 and intron 1 . Sequences in bold represent exons. Sequences in italics are repeats. Putative regulatory sequences are identified as follows: dashed box $=$ retinoic acid response element; underline $=$ E-box, basic helix-loop-helix consensus sequence; solid box = homeodomain, tandem consensus motifs for Drosophila antennapedia, fushi tarazu, and ultrabithorax homeodomain proteins; even dashed line $=$ consensus TATA and CAAT box promoter sequences; uneven dashed line $=$ AP- 2 consensus sequence; dotted line $=$ sequences that show no similarity to known cis-acting regulatory elements, but are similar to sequences in the rat $\alpha 1$ promoter (Gloster et al., 1994).

\section{$\alpha 1-T u b u l i n$ Promoter Activity in Regenerating CNS Neurons}

Explants of adult goldfish retina have been shown to regenerate their ganglion cell axons in tissue culture (Landreth and Agranoff, 1979). To determine if the $\alpha 1$-tubulin promoter was activated during CNS regeneration, we took advantage of the observation that explanted adult goldfish retinas do not extend neurites efficiently unless their optic nerve was crushed in vivo a few days prior to explantation (Landreth and Agranoff, 1976). Quantitation of the number of explants exhibiting outgrowth indicates that an in vivo optic nerve crush can increase the number of explants exhibiting neurite outgrowth by 3 - to 10 -fold. Therefore, we crushed the optic nerve emanating from the right retina of adult goldfish 7 days prior to isolating right and left retinas. Retinas were electroporated with the $\alpha 1$ TIpEGFP expression vector as described in Materials and Methods. Following electroporation, retinas were placed in tissue culture dishes and assayed for reporter gene expression 7 days later. This time point was chosen because it allowed sufficient time for cells to regrow axons which facilitated the identification of regenerating neurons. The number of expressing cells and the level of expression did not appear to decrease from 2 to 7 days following electroporation.

These assays revealed robust expression of $-1696 \alpha 1$ TIpEGFP in cells growing axons (Fig. 7). Interestingly this expression was enhanced when the retina received a prior, in vivo optic nerve crush (Table 2). Deletion of approximately $800 \mathrm{bp}$ from the $5^{\prime}$ end of this promoter $(-808 \alpha 1$ TIpEGFP) reduced overall expression by about $55 \%$ and an additional 

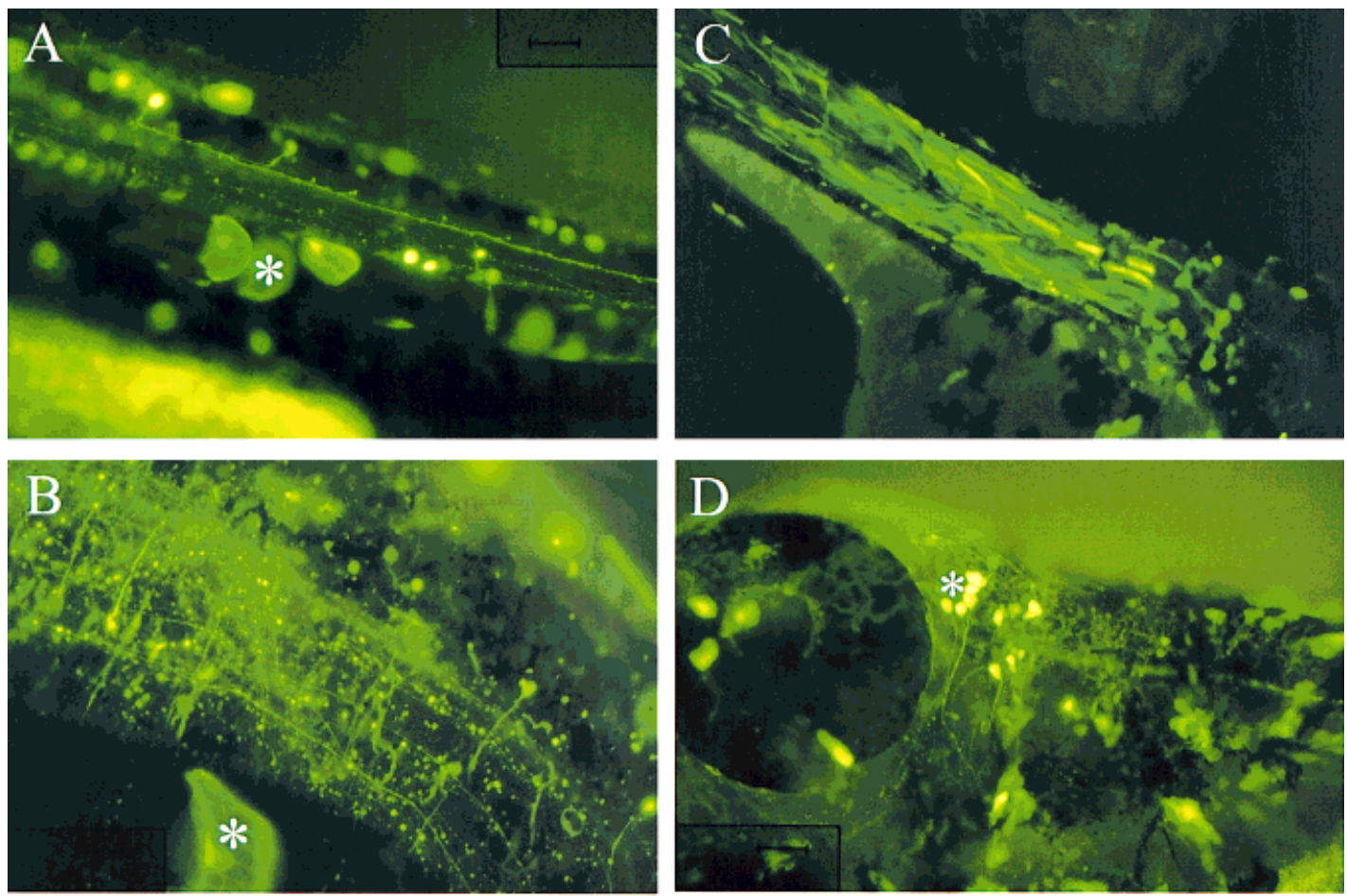

\section{a1 Tubulin}

\section{CMV}

Figure $6 \quad \alpha 1$-Tubulin and CMV promoter-directed expression patterns in developing zebrafish. (A-D) Single-cell zebrafish embryos were injected with either the $-1696 \alpha 1$ TIpEGFP (A,B) or the pCS2 vector (CMV promoter driving GFP expression) (C,D) and examined using fluorescent optics at $48 \mathrm{~h}$ of development. Yellow/green cells and axons represent GFP-expressing cells. (A) Wholemount embryo. Note that many cells are out of the plane of focus, and thus their axons are obscured. The asterisk marks a cluster of three nonneuronal cells expressing the reporter gene. (B) A 48-h zebrafish embryo smashed under a coverslip to get most of the expressing cells in the plane of focus. The asterisk marks a nonneuronal cell expressing the reporter gene. (C) The CMV promoter directs expression to mostly nonneuronal cells in the 48-h zebrafish embryo. (D) The CMV promoter directs expression to both neural and nonneuronal cells. The asterisk in (D) marks a region just behind the eye, where neurons are expressing the reporter gene. Note the $\alpha 1 \mathrm{~T}$ promoter preferentially directs expression to the nervous system, while the CMV promoter does not show neural specificity and is expressed throughout the embryo (see Table 1 for quantitation of these data). Photos were taken with either a $\times 10$ objective $[(C)$, scale bar $=50 \mu \mathrm{m}]$ or a $\times 20$ objective $[(A, B, D)$, scale bar $=100 \mu \mathrm{m}]$.
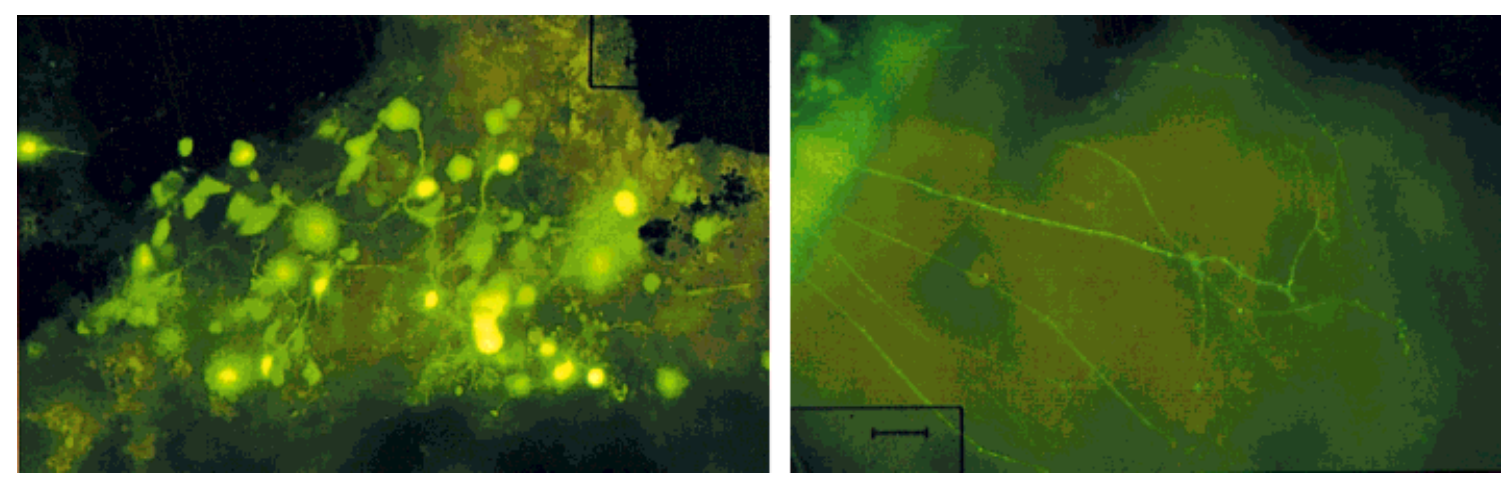

Figure $7-1696 \alpha 1$ TIpEGFP expression in retinal explants. The right optic nerve of adult goldfish was crushed in vivo and retinas isolated 7 days later. Retinas were electroporated with expression vector DNA ( $-1696 \alpha 1$ TIpEGFP) and placed culture for 7 days. Photos show two examples of expression patterns observed in these retinas. Photos were taken with a $\times 20$ objective and fluorescent optics. Scale bar $=100 \mu \mathrm{m}$. 
Table $1 \quad \alpha 1$ ITEGFP Expression in 48-h Zebrafish Embryos

\begin{tabular}{lccr}
\hline Expression Vector & $\begin{array}{c}\text { Embryos Expressing } \\
\text { Injected DNA }\end{array}$ & $\begin{array}{c}\text { Embryos with CNS } \\
\text { Expression (\%) }\end{array}$ & $\begin{array}{c}\text { Expressing Cells } \\
\text { That Are Neural }(\%)\end{array}$ \\
\hline-1696 TI TI pEGFP & 305 & 97 & 82 \\
$-808 \alpha 1$ TI pEGFP & 192 & 82 & 70 \\
$-302 \alpha 1$ TI pEGFP & 234 & 79 & 42 \\
$-182 \alpha 1$ TI pEGFP & 276 & 10 & 8 \\
CMV pEGFP & 64 & 75 & 10 \\
\hline
\end{tabular}

Expression vectors harboring different lengths of the $\alpha 1$-tubulin promoter or the CMV promoter driving GFP expression were injected into one- to two-cell stage embryos and analyzed $48 \mathrm{~h}$ later. Percentage of embryos with CNS expression indicates embryos having at least one neuron expressing the GFP reporter gene. The column reporting percentage of expressing cells that are neural indicates the relative number of GFP expressing cells with a clearly defined axon (at least two cell diameters long) divided by the total number of cells expressing GFP. The data were generated by counting GFP-expressing cells in injected embryos. A minimum of 200 cells were analyzed for each construct.

500-bp deletion (-302 $\alpha 1$ TIpEGFP) had no further effect on expression (Table 2). Nonetheless these constructs were expressed at a higher level (approximately fivefold) in retinas that received a prior in vivo crush (Table 2). Interestingly, an additional deletion of $120 \mathrm{bp}(-182 \alpha 1$ TIpEGFP) abrogated regeneration-dependent induction of this promoter (Table 2). In contrast to the regulated expression of the $\alpha 1$ tubulin promoter, we observed no significant difference between right and left retinas electroporated with an expression vector harboring the CMV promoter driving GFP expression (Table 2). However, the expression of the CMV promoter was much more robust than the $\alpha 1$-tubulin promoter in these retinal explants, consistent with its nonspecific high-level expression pattern observed in vivo [Fig. 6(C,D)].

\section{DISCUSSION}

We report here the cloning and expression of the goldfish $\alpha 1$ - and $\alpha 2$-tubulin genes. These genes are linked in the goldfish genome and were designated $\alpha 1$ - and $\alpha 2$-tubulins based on the similarity of their deduced amino acid sequence with various vertebrate $\alpha$-tubulins (Sullivan, 1988; Ginzburg et al., 1986; Lemischka and Sharp, 1982; Cowan et al., 1983; Lewis et al., 1985; Villasante et al., 1986). The reason for maintaining two very similar tubulin genes whose products differ by a single amino acid is not clear. However, it has been suggested that multiple genes encoding functionally similar proteins may provide an opportunity for multiple independent transcriptional controls (Sullivan, 1988). This is consistent with the observed regulation of the $\alpha 1$-tubulin gene and the constitutive expression of the $\alpha 2$ gene.

Although the $\alpha 2$ gene is expressed in many tissues, it is also expressed in the developing brain where the $\alpha 1$ gene is preferentially expressed (Lewis et al.,
1985). One way to ensure coexpression of two genes is for them to share common cis-acting regulatory elements. For example, regulatory elements located between particular Hox genes are thought to influence the expression of those Hox genes flanking this region of DNA (Sham et al., 1990; Whiting et al., 1991). Therefore, the tight linkage of the $\alpha 1$ - and $\alpha 2$-tubulin genes may also reflect a requirement for shared regulatory elements, perhaps allowing expression of these genes in the nervous system (Lewis et al., 1985). Furthermore, it is likely the $\alpha 2$ gene contains additional regulatory elements mediating its expression in other tissues, while the $\alpha 1$ gene may contain elements that repress its expression in nonneural cells.

Although the goldfish $\alpha 1$ - and $\alpha 2$-tubulin genes encode almost identical proteins that are expressed in neural tissue, they are regulated very differently during CNS development and regeneration (Figs. 2-4). The fish $\alpha 1$-tubulin RNA, like its mammalian counterpart (Miller et al., 1987, 1989), increases at a time when axons are growing (Figs. 2-4). Developmentally, this is illustrated in the retina where $\alpha 1$ gene expression is localized to the middle third, a region enriched in neuroblasts that are differentiating into retinal ganglion cells (Sharma and Ungar, 1980). Within this area of the retina, $\alpha 1$ expression is highest in those cells approaching the inner limiting membrane. Based on this location and their high level of $\alpha 1$-tubulin gene expression, these cells likely represent ganglion cells that are extending axons. The lower level of expression observed throughout the middle third of the developing retina may represent dividing neuroblasts committed to a neuronal fate and/or postmitotic cells migrating to their final position. This expression profile is similar to that of the rat $\mathrm{T} \alpha 1$ promoter driving reporter gene expression in developing mouse retina (Gloster et al., 1994).

In contrast to the regulated expression of the $\alpha 1$ gene, $\alpha 2$-tubulin gene expression does not correlate 
Table $2 \alpha 1$ ITEEFP Expression in Retinal Explants

\begin{tabular}{|c|c|c|c|c|}
\hline \multirow[b]{2}{*}{ Expression Vector } & \multirow{2}{*}{$\begin{array}{l}\text { Retinas Examined } \\
\text { (Right or Left) } \\
(n)\end{array}$} & \multicolumn{2}{|c|}{$\begin{array}{c}\text { Total No. of GFP-Expressing } \\
\text { Cell Clumps }\end{array}$} & \multirow{2}{*}{$\begin{array}{l}\text { GFP-Expressing Cell } \\
\text { Clumps } \\
\text { (Right/Left) }\end{array}$} \\
\hline & & Left Retina & Right Retina & \\
\hline$-1696 \alpha 1$ TI pEGFP & 48 & 36 & 176 & 4.9 \\
\hline$-808 \alpha 1$ TI pEGFP & 24 & 8 & 40 & 5 \\
\hline$-302 \alpha 1$ TI pEGFP & 24 & 8 & 48 & 6 \\
\hline$-182 \alpha 1$ TI pEGFP & 24 & 6 & 8 & 1.3 \\
\hline CMV pEGFP & 12 & 77 & 69 & 0.9 \\
\hline
\end{tabular}

Expression vectors harboring different lengths of the $\alpha 1$-tubulin promoter or the CMV promoter driving GFP expression were electroporated into adult retinas as described in Methods. The optic nerve emanating from the right retina was crushed in vivo 7 days prior to isolating left and right retinas. Positive expressing cells were identified using a Zeiss Axiophot microscope equipped with fluorescent optics. GFP-expressing cells were found in clumps containing anywhere from 1 to 100 cells. We obtained similar results regardless of whether we counted individual cells or clumps of expressing cells. Over $70 \%$ of the expressing cells had a clearly defined axon.

with axon outgrowth (Figs. 2-4). These results are reminiscent of the rat $\mathrm{T} \alpha 1$ and $\mathrm{T} 26$ expression patterns (Miller et al., 1987, 1989), where T $\alpha 1$ is regulated by nerve growth and regeneration and the T26 RNA is constitutively expressed. Although the mouse $\alpha 2$-tubulin RNA has been reported to be expressed in the developing brain, it is expressed at a significantly lower level than the $\alpha 1$-tubulin RNA (Lewis et al., 1985). This lower level of expression may explain why we did not detect this RNA in the developing retina. Alternatively, the $\alpha 2$-tubulin RNA may be induced in the developing retina at a later time. Finally, these results are consistent with our previous reports showing a large increase in $\alpha$-tubulin RNA following optic nerve crush in fish (Hieber et al., 1992; Hieber and Goldman, 1995). However, in those studies, we did not distinguish between $\alpha 1$ - and $\alpha 2$ tubulin isoforms.

The above described increases in $\alpha 1$-tubulin RNA may reflect transcriptional and/or posttranscriptional mechanisms. To explore transcriptional mechanisms, we isolated the $\alpha 1$-tubulin gene's 5'-flanking DNA and assayed its activity during CNS development and regeneration. These experiments showed that a 1696-bp $\alpha 1$-tubulin promoter directs reporter gene expression to the nervous system (Fig. 6 and Table 1). We found that reporter gene expression was highest around 24-48 h of development, corresponding to a time when the nervous system is experiencing extensive axonogenesis (Ross et al., 1992). This pattern of expression was not mimicked by our control CMVpEGFP vector, which was expressed in many cell types located throughout the developing zebrafish.

To confirm that $\alpha 1$-tubulin promoter sequences are necessary for conferring a developmentally regulated, neuron-specific pattern of reporter gene expression, we generated a number of $\alpha 1$ TIpEGFP constructs that contained various promoter deletions (Table 1). In vivo expression assays using these constructs identified two main regulatory regions. First, deletion of sequences between -808 and -302 reduced neuronspecific expression from $70 \%(-808 \alpha 1$ TIpEGFP) (Table 1$)$ to about $42 \%(-302 \alpha 1$ TIpEGFP) (Table 1$)$, yet maintained it above that of the promiscuous CMV promoter (10\%) (Table 1). Second, removal of an additional $120 \mathrm{bp}$ from $-302 \alpha 1$ TIpEGFP to generate $-182 \alpha 1$ TIpEGFP resulted in a large decrease in the number of embryos exhibiting expression in the nervous system (10\% neural) (Table 1) and reduced the percentage of expressing cells that are neurons to $8 \%$. These results suggest that multiple $\alpha 1$-tubulin promoter elements contribute to the expression pattern of this gene.

Although both $-182 \alpha 1$ TIpEGFP- and CMVpEGFPinjected embryos resulted in approximately $8-10 \%$ of the total expressing cells representing neurons (Table 1, column 4), the number of embryos exhibiting neural expression was dramatically different (10\% for $-182 \alpha 1$ TIpEGFP and $75 \%$ of CMVpEGFP) (Table 1 , column 3). These results suggest that the $\alpha 1$-tubulin promoter, containing only $182 \mathrm{bp}$ of $5^{\prime}$-flanking DNA, is a much weaker promoter than the CMV promoter and appears to have lost elements directing its expression to the developing nervous system (i.e., only $8 \%$ of total expressing cells represent neurons) (Table 1).

A number of neural-specific genes have been analyzed for elements mediating their specific expression pattern. These studies have identified a cell typespecific silencer element in the SCG10 and type II sodium channel gene that suppresses expression in nonneural cells (Mori et al., 1990; Kraner et al., 1992). This element was also found in the GAP-43 gene (Nedivi et al., 1992) and contains the core sequence $\mathrm{CCAGG}$. We note that this core sequence 
exists in the $\alpha 1$-tubulin promoter at position -535 and may contribute to the loss of neural specificity observed with $-302 \alpha 1$ TIpEGFP (Table 1). However, there are a number of putative regulatory elements located in the region -808 to -302 (Fig. 5) that may also participate in directing gene expression to the nervous system.

To determine if the cloned fish $\alpha 1$-tubulin promoter is capable of being activated in regenerating CNS tissue, we introduced our $\alpha 1$ TIpEGFP expression vector into retinal explants. For these experiments, we took advantage of the observation that adult goldfish retinas explanted to tissue culture do not regenerate axons efficiently unless their optic nerve was crushed in vivo a few days prior to explantation (Landreth and Agranoff, 1976). These experiments showed that an $\alpha 1$-tubulin promoter containing as little as 302 bp of $5^{\prime}$-flanking DNA was able to increase reporter gene expression in retinas induced to regenerate their neurons (Table 2). However, deletion of an additional $120 \mathrm{bp}(-182 \alpha 1$ TIpEGFP) prevented increased GFP expression in retinas induced to regenerate (Table 2).

It is interesting that both developmentally induced, neuron-specific and regeneration-dependent induction of the $\alpha 1$-tubulin gene required sequences located within a 302-bp region upstream of the $\alpha 1$-tubulin gene's transcription start site (Tables 1 and 2). This result may indicate that nerve regeneration recapitulates development and that common elements may mediate $\alpha 1$-tubulin gene induction during CNS development and regeneration.

Like the fish gene, the mammalian $\alpha 1$-tubulin gene is also induced in developing and regenerating neurons (Gloster et al., 1994). This similar regulation may indicate that conserved elements mediate their expression profiles. Comparison of the fish and rat $\alpha 1$-tubulin promoter sequences identified three regions of conserved DNA extending beyond eight nucleotides. Two of these conserved sequences are located within a 100-bp region just upstream of the transcription start site (dotted underline in Fig. 5). Neither of these sequences resembles previously identified transcription factor consensus binding sites. The third sequence is located 426 and $560 \mathrm{bp}$ upstream of the fish and rat $\alpha 1$-tubulin gene's transcription start sites, respectively. This sequence represents a tandem consensus sequence for homeodomain proteins antennapedia, fushi tarazu, and ultrabithorax (Biggin and Tjian, 1989). We also found an 8-bp consensus retinoic acid response element that is identical in both fish and rat promoters. Both homeobox binding sites and retinoic acid response elements have been implicated to play important roles in nervous system de- velopment (Chisaka et al., 1991; Guillemot et al., 1993; Kaplan et al., 1993) and may also participate in regulating $\alpha 1$-tubulin gene expression. Future experiments will focus on analyzing the functional significance of these putative regulatory elements in the fish system. In addition, we will focus our efforts on identifying those specific DNA sequences mediating developmental and regeneration-dependent induction of the fish $\alpha 1$-tubulin promoter.

The Genbank accession number for the sequence reported here is AF002654. The authors thank Dr. P. Raymond for providing the stage 22 goldfish embryos, Dr. P. Hitchock for use of Picospritzer, Dr. J. Kuwada for help in setting up our zebrafish colony, and Dr. B. Agranoff for helping house goldfish. This work was made possible by the Lucille P. Markey Charitable Trust, and the Mary Hadly Hughes Memorial/DaPuzzos and the Cowen and Company Optic Nerve Research Fund of the Glaucoma Foundation.

\section{REFERENCES}

Biggin, M. D., and TJIAN, R. (1989). Transcription factors and the control of Drosophila development. Trends Genet. 5:377-383.

Chahine, K. G., Baracchini, E., and Goldman, D. (1993). Coupling muscle electrical activity to gene expression via a cAMP-dependent second messenger system. J. Biol. Chem. 268:2893-2898.

Chisaka, O., Musci, T. S., and CAPPECCHI, M. R. (1991). Developmental defects of the ear, cranial nerves and hindbrain resulting from targeted disruption of the mouse homeobox gene Hox-1.5. Nature 355:516-520.

Cowan, N. J., Dobner, P. R., Fuchs, E. V., and Cleveland, D. W. (1983). Expression of human a-tubulin genes: interspecies conservation of $3^{\prime}$ untranslated regions. Mol. Cell. Biol. 3:1738-1745.

Frohman, M. A. (1990). RACE: rapic amplification of cDNA ends. In: PCR Protocols: A Guide to Methods and Applications. M. A. Innis, D. H. Gelfand, J. J. Sninsky, and T. J. White, Eds. Academic Press, New York, pp. 28-38.

Ginzburg, J., Behar, L., Givol, D., and Littauer, U. Z. (1986). The nucleotide sequence of rat $\alpha$-tubulin: $3^{\prime}$-end characteristics and evolutionary conservation. Nucleic Acids Res. 9:2691-2697.

Gloster, A., Wu, W., Speelman, A., Weiss, S., Causing, C., Pozniak, C., Reynolds, B., Chang, E., Toma, J. G., and Miller, F. D. (1994). The T $\alpha 1 \alpha$-tubulin promoter specifies gene expression as a function of neuronal growth and regeneration in transgenic mice. J. Neurosci. 14:7319-7330.

GRAFSTEIN, B. (1991). The goldfish visual system as a model for the study of regeneration in the central nervous system. In: Development and Plasticity of the Visual System, 
Vol. II. J. R. Cronly-Dillon, Ed. Macmillan, London, pp. 190-205.

Guillemot, F., Lo, L. L.-C., Johnson, J. E., Auerbach, A., Anderson, D. J., and Joyner, A. L. (1993). Mammalian achaete-scute homologue 1 is required for the early development of olfactory and autonomic neurons. Cell 75: 463-476.

Heacock, A. M., and Agranoff, B. W. (1982). Protein synthesis and transport in the regenerating goldfish visual system. Neurochem. Res. 7:771-788.

Hieber, V., Agranoff, B. W., and Goldman, D. (1992). Target-dependent regulation of retinal nicotinic acetylcholine receptor and tubulin RNAs during optic nerve regeneration in goldfish. J. Neurochem. 58:1009-1015.

Hieber, V., and Goldman, D. (1993). Cloning and analysis of nicotinic acetycholine receptor gene expression in goldfish retina during optic nerve regeneration. Methods Neurosci. 12:122-138.

Hieber, V., and Goldman, D. (1995). Trans-synaptic regulation of NMDA receptor RNAs during optic nerve regeneration. J. Neurosci. 15:5286-5296.

Kaplan, D. R., Matsumoto, K., Lucarelli, E., and Thiele, C. J. (1993). Induction of trkB by retinoic acid mediates biological responsiveness to BDNF and differentiation of human neuroblastoma cells. Neuron 11:321-331.

Kraner, S. D., Chong, J. A., Tsay, H.-J., and Mandel, G. (1992). Silencing the type II sodium channel gene: a model for neural-specific gene regulation. Neuron 9:3744.

Landreth, G. E., and Agranoff, B. W. (1976). Explant culture of adult goldfish retina: effect of prior optic nerve crush. Brain Res. 118:299-303.

Landreth, G. E., and Agranoff, B. W. (1979). Explant culture of adult goldfish retina: a model for the study of CNS regeneration. Brain Res. 161:39-53.

LemischKa, I., and Sharp, P. A. (1982). The sequence of an expressed rat $\alpha$-tubulin gene and a pseudogene with an inserted repetitive element. Nature 300:330-335.

Lewis, S. A., LeE, M. G.-S., and Cowan, N. J. (1985). Five mouse tubulin isotypes and their regulated expression during development. J. Cell Biol. 101:852-861.

Meng, A., Tang, H., Ong, B. A., Farrell, M. J., and Lin, S. (1997). Promoter analysis in living zebgrfish embryos identifies a cis-acting motif required for neuronal expression of GATA-2. Proc. Natl. Acad. Sci. USA 94:62676272.

Miller, F. D., Naus, C. C. G., Durand, M., Bloom, F. E., and Milner, R. J. (1987). Isotypes of $\alpha$-tubulin are differentially regulated during neuronal maturation. $J$. Cell Biol. 105:3065-3073.

Miller, F. D., Tetzlaff, W., Bisby, M. A., Fawcett, J. W., and Milner, R. J. (1989). Rapid induction of the major embryonic $\alpha$-tubulin mRNA, T $\alpha 1$, during nerve regeneration in adult rats. J. Neurosci. 9:1452-1463.

Mori, N., Stein, R., Sigmund, O., and Anderson, D. J. (1990). A cell type-preferred silencer element that controls the neural-specific expression of the SCG10 gene. Neuron 4:583-594.

Murray, M., and Grafstein, B. (1969). Changes in the morphology and amino acid incorporation of regenerating goldfish optic neurons. Exp. Neurol. 23:544-560.

Nedivi, E., Basi, G. S., Akey, I. V., and Skene, J. H. P. (1992). A neural-specific GAP-43 core promoter located between unusual DNA elements that interact to regulate its activity. J. Neurosci. 12:691-704.

Ross, L. S., PArrett, T., and EASter, S. S. (1992). Axonogenesis and morphogenesis in the embryonic zebrafish brain. J. Neurosci. 12:467-482.

Sham, M. H., Hunt, P., Nonchev, S., Papalopulu, N., Graham, A., Boncinelli, E., and Krumlauf, R. (1990). Analysis of the murine Hox-2.7 gene: conserved alternative transcripts with differential distributions in the nervous system and the potential for shared regulatory regions. EMBO J. 11:1825-1836.

Sharma, S. C., and Ungar, F. (1980). Histogenesis of the goldfish retina. J. Comp. Neurol. 191:373-382.

SkEne, J. H. P. (1989). Axonal growth-associated proteins. Annu. Rev. Neurosci. 12:127-156.

Stuermer, C. A. O., Bastmeyer, M., Bahr, M., Strobel, G., and PASChKE, K. (1992). Trying to understand axonal regeneration in the CNS of fish. J. Neurobiol. 23:537550.

Sullivan, K. F. (1988). Structure and utilization of tubulin isotypes. Annu. Rev. Cell Biol. 4:687-716.

Villasante, A., Wang, D., Dobner, P., Dolph, P., Lewis, S. A., and Cowan, N. J. (1986). Six mouse $\alpha$-tubulin mRNAs encode five distinct isotypes: testis-specific expression of two sister genes. Mol. Cell. Biol. 6:24092419.

Whiting, J., Marshall, H., CoOK, M., Krumlauf, R., Rigby, P. W., Stott, D., and Allemann, R. K. (1991). Multiple spatially specific enhancers are required to reconstruct the pattern of Hox-2.6 gene expression. Genes Dev. 5:2048-2059. 